TecnoLógicas

ISSN-p 0123-7799

ISSN-e 2256-5337

Vol. 21, No. 41, pp. 63-79

Enero-abril de 2018

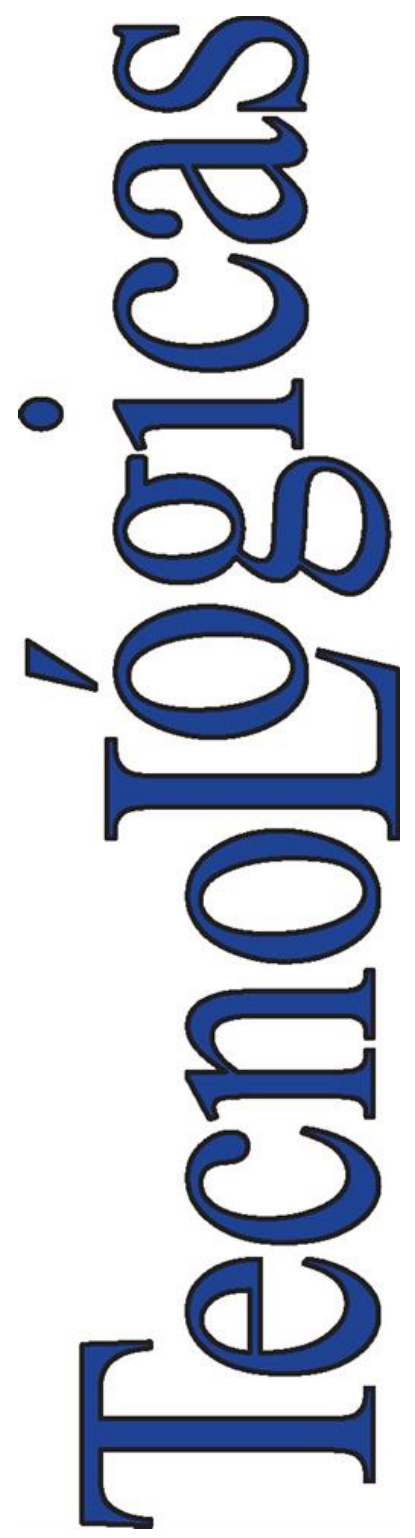

(C) Copyright 2015 por autores y TecnoLógicas Este trabajo está licenciado bajo una Licencia Internacional Creative Commons Atribución (CC BY)

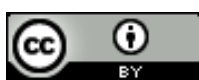

Artículo de Investigación/Research Article

\section{IMU-Mouse: diseño e implementación de un dispositivo apuntador dirigido al desarrollo de interfaces adaptativas para personas con discapacidad física}

\section{IMU-Mouse: design and implementation of a pointing device for people with disabilities}

Camilo A. Castillo-Benavides ${ }^{1}$, Luís F. García-Arias ${ }^{2}$, Néstor D. Duque-Méndez ${ }^{3}$ y Demetrio A. Ovalle-Carranza ${ }^{4}$

Recibido: 06 de junio de 2017

Aceptado: 30 de octubre de 2017

Cómo citar / How to cite

C. A. Castillo-Benavides, L. F. García-Arias, N. D. Duque-Méndez y D. A. Ovalle-Carranza, IMU-Mouse: diseño e implementación de un dispositivo apuntador dirigido al desarrollo de interfaces adaptativas para personas con discapacidad física. TecnoLógicas, vol. 21, no. 41, pp. 63-79, 2018.

1 MSc en Ingeniería - Automatización Industrial, Departamento de Ciencias de la Computación y de la Decisión, Facultad de Minas, Universidad Nacional de Colombia, Medellín-Colombia, cacastilloben@unal.edu.co

2 Ingeniero Electrónico, Universidad Nacional de Colombia, ManizalesColombia, lufgarciaar@unal.edu.co

3 PhD en Ingeniería - Sistemas e Informática, Departamento de Informática y Computación, Universidad Nacional de Colombia, Manizales-Colombia, ndduqueme@unal.edu.co

4 PhD en Informática, Departamento de Ciencias de la Computación y de la Decisión, Facultad de Minas. Universidad Nacional de Colombia, MedellínColombia,dovalle@unal.edu.co 
IMU-Mouse: diseño e implementación de un dispositivo apuntador dirigido al desarrollo de interfaces adaptativas para personas con discapacidad física

\title{
Resumen
}

La aplicación adecuada de las TIC (Tecnologías de la Información y Comunicación) para el aprendizaje de personas con discapacidad física exige estudios dirigidos a la adaptación de interfaces. El control sobre el diseño e implementación de dispositivos de entrada contribuiría al desarrollo de interfaces capaces de atender las necesidades en el contexto descrito anteriormente. El objetivo de este trabajo es presentar el desarrollo y la validación del dispositivo apuntador IMU-Mouse, para el cual se tiene el control sobre su diseño e implementación dirigido a la investigación de interfaces, que hagan uso de las capacidades de interacción del aparato en los procesos de adaptación. El dispositivo está dirigido a atender las necesidades inducidas por la discapacidad física en extremidades superiores y por esto opera con los movimientos de la cabeza. Las acciones de clic y desplazamiento del IMU-Mouse se ejecutan a partir del procesamiento de los datos generados por un acelerómetro y un giroscopio. Los principales aspectos de la interacción fueron evaluados por un grupo de 13 personas mediante una encuesta basada en el anexo C del estándar ISO 9241-9. De acuerdo con los resultados obtenidos, se concluye que el dispositivo habilita una interacción efectiva con el computador, y además permiten la identificación de espacios para la aplicación de complementos de hardware y software para asistir las acciones de interacción.

\section{Palabras clave}

Accesibilidad, discapacidad física, interfaces adaptativas, mouse, unidad de medida inercial.

\begin{abstract}
The correct application of ICTs (information and communications technologies) to the education of people with physical disabilities requires studies aimed to adapt interfaces. Controlling the design and implementation of input devices would contribute to develop interfaces tailored to the requirements of such context. The purpose of this work is to present the development and validation of the IMU-Mouse pointer device whose design and implementation is controlled-for researching interfaces that make the most of the interaction capabilities of the devices in adaptation processes. The purpose of the device is to address the needs created by physical disability in upper limps; hence it operates with head movements. Both actions of the IMU-Mouse, click and move, are performed by processing the data generated by an accelerometer and a gyroscope. The main interaction features of the IMU-Mouse were assessed by group of 13 people by means of a survey based on Annex C of ISO 9241-9 standard. The results lead to conclude that the device enables an effective interaction with the computer, as well as the identification of spaces for the application of hardware and software add-ons to support interaction.
\end{abstract}

\section{Keywords}

Accessibility, physical disability, adaptive interfaces, mouse, inertial measurement unit. 
IMU-Mouse: diseño e implementación de un dispositivo apuntador dirigido al desarrollo de interfaces adaptativas para personas con discapacidad física

\section{INTRODUCCIÓN}

La aplicación de las TIC (Tecnologías de la Información y Comunicación) dirigida al aprendizaje de las personas con discapacidad física exige la ejecución de estudios de usabilidad de dispositivos, herramientas y software. Esta necesidad surge porque se requiere el desarrollo de interfaces que se adapten a las necesidades de este tipo de usuarios desde las áreas de adaptación de contenido, de presentación, de interacción y de navegación [1]. En el área de la interacción se ubican los dispositivos de entrada que brindan accesibilidad a personas con discapacidad física, principalmente mediante la transformación de energía mecánica en energía eléctrica, p. ej., el mouse o el teclado [2]. Para las investigaciones de usabilidad en contextos educativos soportados por TIC, se requiere controlar los aspectos de diseño e implementación de los dispositivos mencionados. La ausencia de este control genera limitaciones en las investigaciones, porque estos aspectos repercuten en las áreas que cubren el desarrollo de interfaces de usuario.

El diseño e implementación de los dispositivos contribuiría a mejorar la construcción de interfaces que se adapten al usuario y que estén orientadas a la enseñanza. La mejoría se debe a que, además de usar el dispositivo para vencer las barreras para la interacción, también se pretende alcanzar un grado adecuado en usabilidad dirigido al aprendizaje mediante el uso de recursos educativos digitales [3]. Un nivel adecuado en usabilidad contribuye a disminuir la marginación de las personas con Necesidades Especiales en la Diversidad (NEED), originada principalmente por la interacción de estudiantes con limitaciones físicas mediante interfaces de usuario generalizadas. El problema con estas interfaces de uso extendido es que además de su baja capacidad de adaptación usualmente se implementan a partir de suposiciones tales como las siguientes: se asume que el usuario no presenta ningún tipo de disca- pacidad, están diseñadas para ser usadas con un conjunto estándar de dispositivos de interacción, y se supone que el entorno que rodea al individuo no impone ningún tipo de barrera [4].

Mantener el control sobre el diseño e implementación de los dispositivos constituye un reto debido a que distintas disciplinas contribuyen en cada una de las áreas de adaptación [1]. El mejoramiento de la usabilidad abordada desde el área a la que pertenece cada profesional constituye en sí mismo un esfuerzo en investigación. Por ejemplo, los aportes podrían estar dirigidos a la adaptación de contenido educativo, a la adecuación del tamaño de las fuentes, o a la definición de los posibles caminos que el usuario puede seguir para alcanzar la información dentro de una aplicación [1], apartando así la adaptación de componentes de hardware y los elementos de software asociados a estos. Dejar de lado estos aspectos implica que se generen vacíos en la usabilidad global de las interfaces, porque no se tienen en cuenta las características de los dispositivos de entrada para la adaptación. La importancia de incluir estas características podría ser expuesta mediante un caso en donde un profesional intente aportar a la usabilidad desde el área de presentación; el recurso utilizado para dicho aporte estaría basado en el agrandamiento de los botones en la pantalla. La medida del agrandamiento sería controlada a partir de la posibilidad de asistir la ejecución de las tareas de interacción (p. ej., las tareas de clic, señalar, arrastrar, teclear, entre otras) [5], es decir, con ayuda de una estrategia de adaptación basada en hardware.

A partir de una revisión previa de la literatura, presentamos como ejemplos más representativos los siguientes sistemas adaptativos reportados en el informe del proyecto MyUI [1]: Doppel-gänger, Flexcel, Lifestyle Finder, ELM-ART II, LumiEreProject, Personal Universal Controller, SUPPLE, Huddle, UNIFORM, AUGUR, sistema de aprendizaje adaptativo con LS- 
IMU-Mouse: diseño e implementación de un dispositivo apuntador dirigido al desarrollo de interfaces adaptativas para personas con discapacidad física

Plan, sistema de realidad aumentada para el aprendizaje móvil adaptativo basado en la ubicación del usuario y SmartCal. Otras propuestas de enfoques adaptativos son: sistema de agentes inteligentes en un entorno e-learning [6], método híbrido para un sistema de recomendación de objetos de aprendizaje [7]. Una de las características que comparten los sistemas mencionados es que las adaptaciones realizadas sobre las interfaces están concentradas en las áreas de contenido, navegación o presentación. Como excepción es resaltado el proyecto MyUI [1], en donde son tenidas en cuenta todas las áreas de adaptación mencionadas. La limitante que se encuentra consiste en que los dispositivos no hacen parte activa de la adaptación porque son incluidos mediante descripciones basada en pares de nombre y valor [8], [9]. Al dejar de lado la capa de interacción se abandona la posibilidad de transformar a los dispositivos de entrada en componentes activos para la adaptación. Esta propuesta está dirigida al desarrollo de esta transformación mediante la asistencia a las tareas de interacción según las necesidades del usuario.

Las principales soluciones reportadas en lo que respecta a la interacción de personas con discapacidad física mediante dispositivos apuntadores son: joysticks, mouthsticks, trackballs, pantallas táctiles, reconocimiento de gestos (p. ej., gestos de las manos, de la cara, o de los ojos), sensores inerciales, interfaces cerebrocomputador, comandos de voz o soplidos, señales eléctricas de los músculos, y dispositivos basados en interruptores [10], [11]. La mayoría de tecnologías de asistencia para la interacción -en donde también se incluyen a los dispositivos apuntadores basados en las soluciones mencionadas- no solventan el problema relacionado con la inserción de las TIC en la comunidad con discapacidad [12]. Según Kouroupetroglou [12], el problema con estas tecnologías son los precios elevados, las limitadas capacidades de los aparatos según las funcionali- dades que pueden desempeñar, la complejidad para ser configurados, y el tiempo de aprendizaje que se requiere para ser operados. A partir de lo anterior se infiere que, el desarrollo de los dispositivos debe estar centrado en explotar las fortalezas de cada una de las soluciones manteniendo la calidad en lo que respecta a la usabilidad.

En este artículo se presenta el desarrollo de un dispositivo apuntador llamado IMU-Mouse el cual está dirigido a personas con discapacidad física en extremidades superiores como una herramienta de investigación para el desarrollo de interfaces. Con base en el estándar ISO 9241-9 se consultaron 13 personas con el fin de evaluar el potencial del IMU-Mouse como dispositivo apuntador. Debido a que se tiene dominio sobre el desarrollo del aparato, la consulta también fue utilizada para la identificación de espacios de aplicación de complementos de hardware y software para asistir las tareas de interacción. Los complementos servirían como elementos útiles para la investigación de modelos de adaptación de interfaces en el área de interacción que estén acoplados al resto de las áreas. Los resultados obtenidos permitieron concluir que el IMU-Mouse permite realizar una interacción efectiva y al mismo tiempo identificar los espacios de aplicación de complementos en función de las siguientes limitaciones: dificultad para seleccionar los objetivos de las esquinas, cansancio inducido por el golpeteo del clic, y falta de precisión del clic debido a su dependencia con la acción de desplazamiento. Así se infiere que los complementos a considerar deberán ser aquellos que, mediante software o hardware, permitan mejorar la precisión o independicen el clic de la acción de desplazamiento (p. ej., el uso de señales del cerebro [13] o el uso de un micrófono [14]).

Es preciso señalar que en la literatura se reportan otras propuestas que integran una IMU y tienen el potencial para desempeñarse como un mouse operado con la cabeza [15] y [23]. Las principales desven- 
IMU-Mouse: diseño e implementación de un dispositivo apuntador dirigido al desarrollo de interfaces adaptativas para personas con discapacidad física

tajas que comparten con el dispositivo propuesto en este documento son la ausencia de un diseño adecuado de la diadema y las limitaciones existentes para la ejecución del clic y sus derivados. Ninguno de los aparatos reportados fue utilizado como una herramienta de investigación para el desarrollo de interfaces adaptativas en el campo del hardware. Esto implica que se dificulte la integración a estos dispositivos con otras características adicionales de software y hardware para atender las necesidades particulares de algún individuo.

Aunque el IMU-Mouse está dirigido a la interacción de usuarios con ausencia de extremidades superiores, el objetivo de este también se centra en servir como una herramienta para el desarrollo de dispositivos que se incluyan en procesos de adaptación de interfaces. Tanto el tipo de diseño como el de implementación seleccionados facilitarían la explotación de las soluciones reportadas en la literatura, mientras se atiende la usabilidad. El diseño basado en medidas inerciales ofrece múltiples ventajas para atender las necesidades relacionadas con el desplazamiento del cursor tales como: los tiempos de aprendizaje se reducen debido a que se puede asociar el desplazamiento y los movimientos que el usuario puede ejecutar, se libera al usuario de la necesidad de mantener una posición determinada con respecto a la pantalla como ocurre con los sistemas basados en gestos, la complejidad de configuración recae principalmente en los desarrolladores -tanto que en este caso el usuario únicamente debe conectar el dispositivo a través de un puerto USB para comenzar a utilizarlo-, y el valor de los sensores es reducido en comparación con las otras propuestas. Teniendo en cuenta que el enfoque basado en medidas inerciales presenta limitaciones en lo que respecta a la precisión del clic, la implementación basada en la tarjeta de desarrollo Arduino ofrece opciones para tomar ventaja de las soluciones encontradas mediante su aplicación. Por ejemplo, en el mercado se encuentran tarjetas complementarias para el reconocimiento de voz o para el uso de señales BCI tales como el módulo Elechouse V3 o el chip ThinkGear AM de Neurosky. Estas tarjetas son muestras de lo que en este documento se llaman complementos, los cuales tienen la finalidad de enriquecer la interacción.

En los párrafos anteriores se ha establecido el contexto de investigación en el desarrollo del IMU-Mouse y presentado los trabajos relacionados. El resto del documento se compone de la siguiente manera: en la sección materiales y métodos se dan a conocer los principios conceptuales que rigen el funcionamiento de los sensores de la Unidad de Medida Inercial (IMU, por su sigla en inglés), se definen los gestos de interacción en función de los movimientos de la cabeza, se presenta la integración de los gestos con el uso de los sensores, y se describe la construcción del dispositivo haciendo uso de un IMU GY-85 y un Arduino Pro Micro. En la sección planteamiento del experimento se presenta el diseño de una prueba que busca evaluar el dispositivo como herramienta de interacción y el procedimiento a seguir para su ejecución. En la sección resultados y discusión se inicia con la validación acerca del diseño del funcionamiento de dispositivo, luego se presentan los resultados del experimento junto con una discusión dirigida a resaltar los principales hallazgos obtenidos. Finalmente se presentan las secciones de conclusiones y de trabajo futuro.

\section{MATERIALES Y MÉTODOS}

\subsection{Sensores}

En esta sección se desarrolla el diseño de un dispositivo de interfaz humana basado en una unidad de medida inercial (IMU) [24]. Esta unidad permite conocer los datos de posición y desplazamiento de un sistema porque integra un conjunto de sensores que permiten conocer condiciones 
IMU-Mouse: diseño e implementación de un dispositivo apuntador dirigido al desarrollo de interfaces adaptativas para personas con discapacidad física

de aceleración, velocidad angular, y en algunos casos orientación relativa al campo magnético terrestre. Como conjunto, las mediciones obtenidas de los sensores incluidos en las IMU tienen aplicaciones en los sistemas de navegación inercial como los usados en aeronaves, misiles o sistemas robóticos autónomos [24]. En este caso, la información obtenida permite que usuarios con dificultades en extremidades superiores ejecuten las acciones de clic y desplazamiento del cursor.

Las IMU convencionales integran un acelerómetro y un giroscopio, aunque pueden incluir sensores adicionales tales como magnetómetros. Un acelerómetro mide las fuerzas de inercia que actúan sobre un cuerpo [25]. Los datos obtenidos con este sensor permiten al IMU-Mouse activar o desactivar la interacción con el sistema operativo y asistir la detección del clic. Un giroscopio consiste en un cuerpo rígido que es acoplado a un marco móvil para permitir el cambio de inclinación del eje de rotación del cuerpo. Un cambio de posición angular genera un momento que puede ser cuantificado midiendo la trayectoria que describe el marco móvil acoplado sobre la masa inercial [26]. Los datos obtenidos a partir del giroscopio complementan la información del acelerómetro para desplazar el cursor y reconocer el clic.

\subsection{Definición de acciones de interacción}

En la Fig. 1 se ilustran las acciones de interacción implementadas en el dispositivo: desplazamiento del cursor y clic. Para la acción de desplazamiento se desea que el usuario relacione de manera intuitiva los movimientos de la cabeza con los movimientos del cursor (Fig. 1.a). El desplazamiento horizontal se obtiene a través del desplazamiento angular sobre el eje transversal de la cabeza. De manera análoga, se logra el movimiento vertical del cursor a partir del desplazamiento angular de la cabeza sobre su eje longitudinal. De este modo, el dispositivo identifica el desplazamiento de la cabeza y lo relaciona de manera lineal con la magnitud de desplazamiento en pixeles para ejecutar un desplazamiento relativo en pantalla. Para la acción de clic se seleccionó un movimiento que consiste en generar un cambio marcado de aceleración mediante un movimiento que consiste en asentir con la cabeza (Fig. $1(b))$.
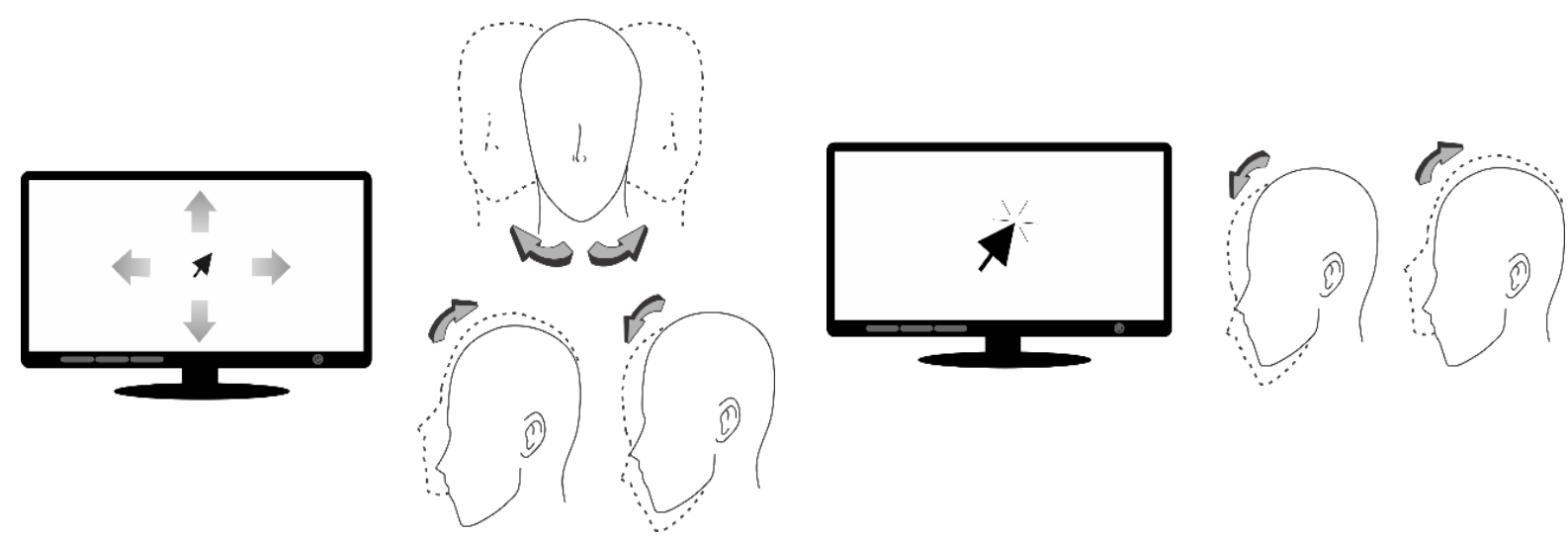

(a)

(b)

Fig. 1. Acciones de Interacción. (a) Desplazamiento del cursor. (b) Clic. Fuente: autor. 
IMU-Mouse: diseño e implementación de un dispositivo apuntador dirigido al desarrollo de interfaces adaptativas para personas con discapacidad física

\subsection{Implementación}

Como sistema de medida del mouse, se utiliza la tarjeta IMU GY-85 que posee un acelerómetro digital ADXL-345, un giroscopio ITG-3205 y un magnetómetro HMC5853L. La placa Arduino Pro Micro se selecciona como sistema de procesamiento debido a que posee un amplio conjunto de librerías basadas en $\mathrm{C}$, que reducen los tiempos de desarrollo. Los sistemas se ubican sobre una diadema para que el dispositivo permanezca estable en la cabeza del usuario (Fig. 2.a). En Fig. 2.b se presenta la implementación física del dispositivo: la integración de la IMU y el Arduino sobre un circuito impreso, la caja contenedora del circuito con un orificio que permite la conexión micro USB, y el dispositivo ubicado sobre la cabeza de un usuario sin brazos. El peso en gramos de la placa que contiene los componentes electrónicos es de $12,76 \mathrm{~g}$, el cable USB que une el dispositivo desde la cabeza hasta el computador pesa $31.68 \mathrm{~g}$, y el peso total del dispositivo es de 132.86 g. Desde el punto de vista eléctrico, el IMU-Mouse no pone en peligro la seguridad del usuario porque sus componentes se encuentran aislados de la piel.

En Fig. 2a se muestra la disposición de los ejes de respuesta del acelerómetro ADXL-345 y del giroscopio ITG-3205 según la ubicación de la IMU en la diadema. En el ADXL-345 las variaciones de aceleración son medidas a lo largo de los ejes $\mathrm{x}, \mathrm{y}$ e $\mathrm{z}$. Cuando el cambio de aceleración se produce en la dirección de los ejes se toma como positiva, en caso contrario se toma como negativa. Para la aceleración se usa como unidad de medida la fuerza g, en donde $1 \mathrm{~g}$ equivale a la gravedad terrestre (9.806 $\mathrm{m} / \mathrm{s} 2$ ). El ITG-3205 entrega tres medidas de la velocidad con la cual cada uno de los ejes $\mathrm{x}, \mathrm{y}$ e $\mathrm{z}$ rotan sobre sí mismos. Las mediciones están dadas en grados sobre segundo $(\%)$ y para este caso se toman como positivas cuando los movimientos están a favor de las manecillas del reloj.

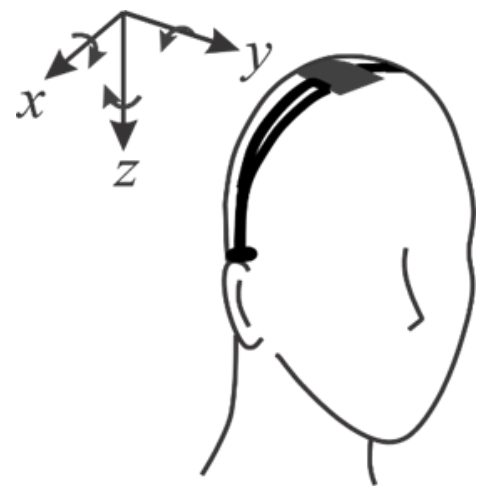

(a)

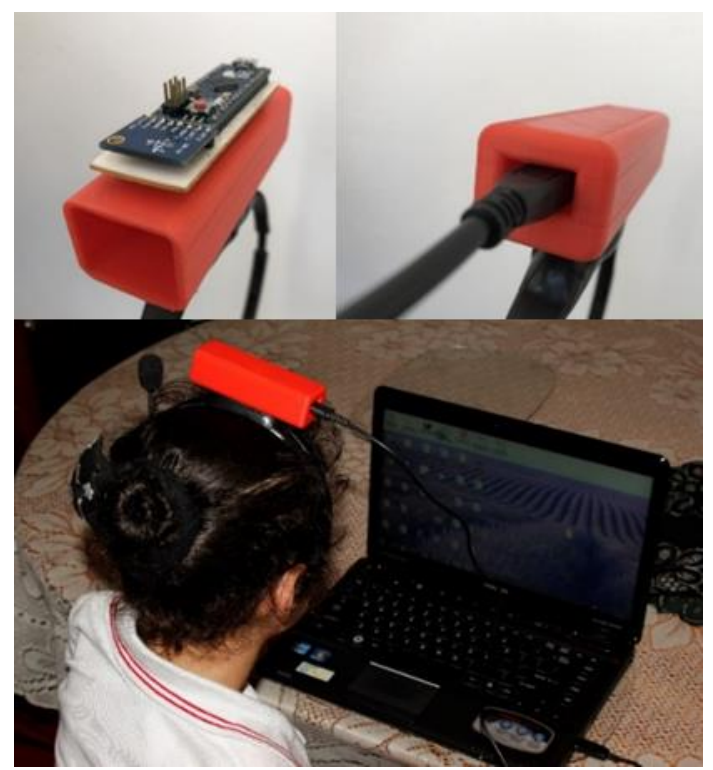

(b)

Fig. 2. Dispositivo IMU-Mouse. (a) Ubicación del sistema sobre la diadema y disposición de los ejes primarios del acelerómetro y giroscopio. (b) Implementación física del dispositivo. Fuente: autor y foto de Andrés Almeida (agencia de noticias Universidad Nacional de Colombia, 20 de mayo de 2016). 
IMU-Mouse: diseño e implementación de un dispositivo apuntador dirigido al desarrollo de interfaces adaptativas para personas con discapacidad física

\subsection{Funcionamiento del dispositivo}

La interpretación de las acciones de interacción por parte del sistema de procesamiento se basa en el aprovechamiento de las características del sistema de medida IMU GY-85. Entre los parámetros configurados en la unidad de medida se destacan los rangos de sensibilidad, los eventos de actividad e inactividad basados en el acelerómetro, y la frecuencia de corte del filtro pasa bajas digital del giroscopio. El rango de medida del acelerómetro se configuró en $\pm 4 \mathrm{~g}$ con un tamaño de paso especificado en la hoja de datos de $1.95 \mathrm{mg} / \mathrm{LSB}$. El rango del giroscopio se estableció en $\pm 2000 \%$ s con un tamaño de paso especificado en la hoja de datos de $14.375 \mathrm{LSB} / \%$ s. Los eventos de actividad e inactividad se implementan mediante la programación de interrupciones de hardware y se activan cuando se alcanzan umbrales de aceleración definidos para cada uno. Las librerías utilizadas para la configuración de la IMU y el procesamiento de la información fueron: Wire.h, I2Cdev.h, ADXL345.h, HMC5883L.h, ITG3200.h, y TimerOne.h.

Las señales del acelerómetro y del giroscopio pueden verse afectadas por las variaciones de posición de la cabeza inherentes al equilibrio del cuerpo, a factores de ruido propios de los sensores e incluso a variaciones de temperatura. Se considera importante reducir los efectos de estas interferencias para evitar comportamien- tos indeseados del puntero sobre la pantalla. Para reducir estos efectos se establecen umbrales de actividad e inactividad, basados en valores de aceleración que definen tres zonas de funcionamiento: zona de inactividad, zona de transición y zona de actividad (Fig. 3a). Después de la activación, se utilizan los datos de velocidad angular para desplazar el puntero durante el tiempo en que el dispositivo permanece en zona de actividad y de transición (Fig. 3b). La zona de transición constituye un límite dinámico de tal manera que el sistema entra en actividad para un valor de aceleración que sobrepasa $0.19 \mathrm{~g}$, y regresa a la inactividad para un valor que cae por debajo de $0.13 \mathrm{~g}$. Los valores se fijaron después de distintos ensayos buscando que el proceso de activación no cause una fatiga significativa en el cuello mientras se reducen los efectos de las interferencias externas. Para que suceda el evento de actividad se debe superar el umbral de $0.19 \mathrm{~g}$ en cualquiera de los ejes $x$ e $y$; el evento de inactividad ocurre si el valor de la aceleración cae por debajo de $0.13 \mathrm{~g}$ en los dos ejes mencionados. Con el fin de mejorar la robustez del sistema también se fijó en $5 \mathrm{~Hz}$ la frecuencia de corte del filtro pasa bajas del giroscopio. Se estableció este valor teniendo en cuenta que para una aplicación que se basa en los movimientos de la cabeza es conveniente filtrar las componentes de frecuencias mayores.

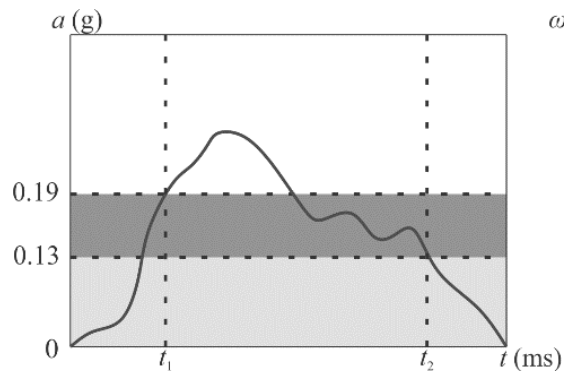

(a)

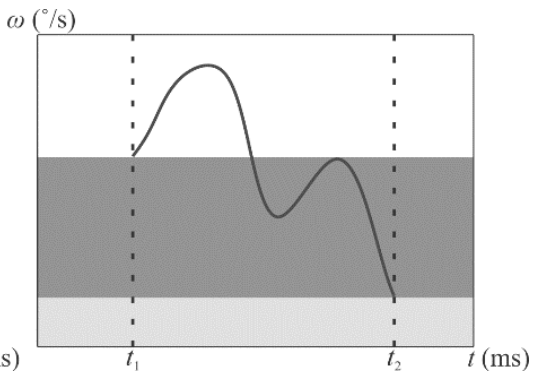

(b)

$\square$ Inactividad $\square$ Actividad $\square$ Transición

Fig. 3. Representación conceptual de la transición entre las zonas de actividad e inactividad. (a) Umbrales de aceleración que delimitan la zona de transición. (b) Los datos de la velocidad angular para ejecutar la acción de desplazamiento se toman en las zonas de actividad y de transición después de la activación. Fuente: autor. 
IMU-Mouse: diseño e implementación de un dispositivo apuntador dirigido al desarrollo de interfaces adaptativas para personas con discapacidad física

Para ejecutar la acción de clic se hace uso conjunto de los datos obtenidos por el acelerómetro y el giroscopio. En este caso se usan los valores de aceleración obtenidos en los ejes y e z para calcular la magnitud de su suma vectorial, es decir, $\left|\boldsymbol{a}_{y z}\right|=$ $\sqrt{\left(a_{y}\right)^{2}+\left(a_{z}\right)^{2}}$. Es preciso señalar que el valor de la magnitud ronda el valor de $1 \mathrm{~g}$ debido a que en el eje $z$ se mide continuamente la aceleración estática de la gravedad terrestre. Los datos del giroscopio se utilizan para detectar cambios acentuados de la velocidad angular, definidos como el valor absoluto de la diferencia entre la muestra actual del sensor y la muestra anterior a esta $|\Delta \omega|$. El asentir con la cabeza implica un desplazamiento angular en el eje $x$, donde son medidos los cambios acentuados de velocidad angular. En Fig. 4 se representa el clic a partir de la forma conceptual de las señales de magnitud y de cambios de velocidad angular originadas cuando se asienta con la cabeza. La ejecución del gesto produce dos cambios marcados en la magnitud de la suma vectorial y del $|\Delta \omega|$. Al ser identificada la acción de clic se eliminan los datos de velocidad angular y el sistema pasa a estado de inactividad.

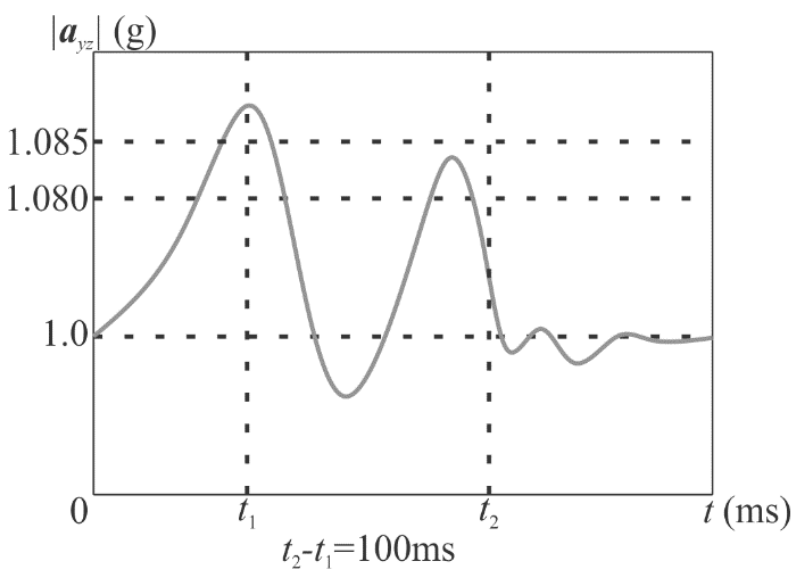

(a)
Los dos cambios mencionados en el párrafo anterior se deben a que, en el proceso de asentir se produce un cambio marcado en las variables cuando la cabeza se desplaza sobre su eje longitudinal en dirección hacia abajo, luego la cabeza desacelera hasta quedar quieta por un momento, y por último se genera el segundo cambio acentuado cuando la cabeza acelera nuevamente sobre su eje longitudinal pero esta vez hacia arriba. La placa Arduino Pro Micro detecta el primer cambio marcado cuando de manera simultánea el valor de la magnitud de la suma vectorial es mayor o igual a $1.085 \mathrm{~g}$ y $|\Delta \omega|$ es mayor o igual a $11 \%$ s. El segundo cambio ocurre cuando de manera simultánea el valor de la magnitud de la suma vectorial es mayor o igual a $1.08 \mathrm{~g} \mathrm{y}$ $|\Delta \omega|$ es mayor o igual a $10 \%$ s. Después de la detección del primer cambio el sistema concede $100 \mathrm{~ms}$ a la espera del segundo. Los umbrales de la magnitud y de la diferencia de la velocidad se establecieron mediante ensayos con el fin de, distinguir el clic del desplazamiento y de procurar la comodidad del usuario. El tiempo de 100 ms también se fijó mediante ensayos en búsqueda del equilibrio entre la comodidad y la necesidad de asentir rápidamente.

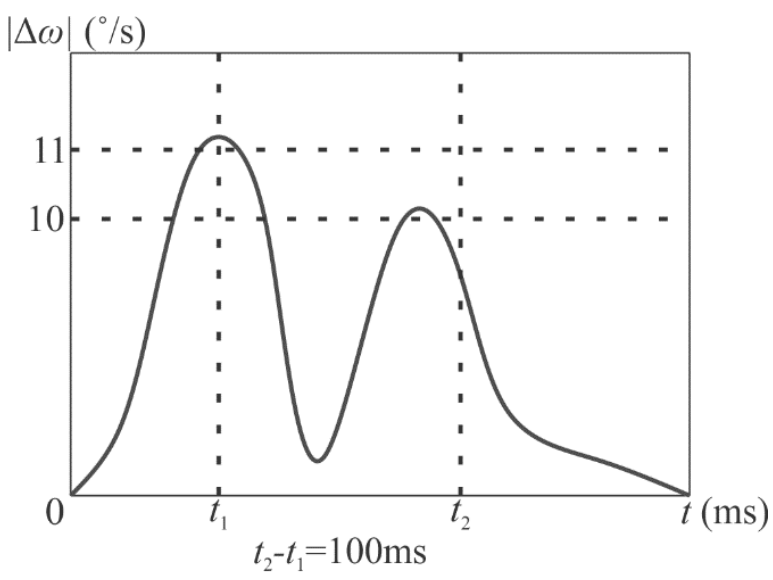

(b)

Fig. 4. Representación conceptual de la ejecución del clic. (a) Cambios en la magnitud $\left|\boldsymbol{a}_{y z}\right|$ de la suma de los vectores de aceleración en los ejes y e z. (b) Cambios en $|\Delta \omega|$ Fuente: autor. 
IMU-Mouse: diseño e implementación de un dispositivo apuntador dirigido al desarrollo de interfaces adaptativas para personas con discapacidad física

\section{Planteamiento del eXPerimento}

Además de permitir la interacción de los usuarios con la computadora, el IMUMouse fue concebido como una herramienta para el desarrollo de complementos de hardware y software que asistan la ejecución de las tareas de un dispositivo apuntador. El experimento está constituido por un cuestionario dirigido a evaluar algunos aspectos de interacción. Los aspectos de consulta incluidos dentro del cuestionario están relacionados con la fatiga, la calidad de la interacción, el esfuerzo, la selección de objetivos y las preferencias. Con base en la información obtenida se pretende inferir algunos espacios para la aplicación de los complementos. El uso de complementos tiene como finalidad reducir las barreras en interacción que podrían surgir a partir del uso de tecnologías asistivas, tales como el IMU-Mouse. En este caso las barreras podrían existir debido a que el dispositivo desarrollado opera con base en los movimientos de la cabeza en lugar de los de la mano.

El cuestionario se complementó mediante dos preguntas enfocadas en la recolección de información adicional sobre el IMU-Mouse. La primera indaga acerca de la posibilidad que tiene el dispositivo para funcionar como una alternativa para individuos con limitaciones físicas en las extremidades superiores. La otra pregunta consiste en la captura de las sugerencias de los participantes mediante la consulta acerca de la calidad general de la interacción. A partir de las sugerencias obtenidas serán planteadas mejoras al proceso de interacción y al diseño del prototipo.

\subsection{Participantes}

La prueba fue ejecutada por un grupo de 13 personas compuesto por cuatro de sexo femenino y nueve de sexo masculino. La edad promedio de la población es de 24,6 años con edades que varían entre los 17 y los 44 años. De los 13 participantes tres personas reportaron situación de discapacidad. Entre las discapacidades se encuentran: físico motora debido a cuadriplejía espástica con reducción del $88.7 \%$ de la movilidad corporal, cuadriplejía por lesión raquimedular, y amputación de extremidades superiores a nivel de muñeca en la extremidad izquierda y a nivel de falanges proximales en extremidad derecha. El promedio de uso del computador es de 5,4 horas en un rango en horas entre $1 \mathrm{y}$ 13,5 por día. Tres de los 13 encuestados usan el panel táctil para interactuar con el computador y los 10 participantes restantes utilizan el mouse convencional incluyendo a quienes reportaron situación de discapacidad.

\subsection{Equipamiento}

El equipamiento consiste en un equipo con sistema operativo Windows 10 , en el que se ejecuta la prueba propuesta. La prueba se hace en un entorno uniforme para los participantes debido a que la interacción con el dispositivo puede ser influenciada por la distancia del usuario a la pantalla, la resolución y el tamaño. La pantalla utilizada posee una resolución de 1024 x 768 píxeles y un tamaño de 19 pulgadas. Las personas que reportan discapacidad motora utilizaron sus propios equipos debido a la dificultad que implica replicar las mismas condiciones en sus respectivas viviendas.

\subsection{Procedimiento}

Siguiendo a [27] y [28], el procedimiento de evaluación fue dividido en cuatro partes. Al principio del proceso se recopila la información general del participante de la prueba. Luego se realiza una sesión de práctica con el objetivo de familiarizar al usuario con la herramienta. Seguidamente, se procede con la sesión de prueba basada en la selección de objetivos en la pantalla mediante las tareas de desplazamiento y clic. Por último, los resultados son obteni- 
IMU-Mouse: diseño e implementación de un dispositivo apuntador dirigido al desarrollo de interfaces adaptativas para personas con discapacidad física

dos a partir de una encuesta acerca de la interacción durante el uso del dispositivo.

La recopilación de información realizada al principio de la prueba inicia con la captura de datos demográficos en donde se indaga acerca de la presencia de algún tipo de discapacidad y su descripción. Continúa la sesión de práctica que tiene como objetivo la familiarización del usuario con el IMU-Mouse. Siguiendo a [29], la tarea de práctica es presentada a los participantes de la prueba como un escenario que los reta a la selección de metas. La posición de una meta será elegida de manera aleatoria para que sea alcanzada con el cursor y luego seleccionada mediante un clic. El entorno de ensayo está constituido por cinco objetivos circulares ubicados en cada una de las cuatro esquinas y el centro de la pantalla. Para el entorno de la prueba se fija la distancia que separa a las metas de los extremos con la meta del centro en 500 píxeles, y el diámetro en píxeles de todas las metas para cada elección aleatoria puede variar entre 75 o 100 . Al inicio de la sesión una persona muestra al usuario la manera en que debe interactuar con el entorno durante el tiempo de ensayo.

Dentro del entorno de interacción es presentada una prueba orientada a la medición del rendimiento de un usuario que hace uso de un dispositivo apuntador. El entorno fue construido con base en los lineamientos establecidos en el anexo B del estándar ISO 9241-9 que evalúa el rendimiento mediante una variación del índice de Fitts [30].

Aunque el test está enfocado en la consulta de algunos aspectos de la interacción, la construcción de la interfaz de prueba se hace con la finalidad de generar un espacio para una futura evaluación cuantitativa del rendimiento del IMU-Mouse y sus posibles complementos. La prueba consiste en seleccionar con el cursor una secuencia de metas presentadas en diferentes excentricidades con respecto al centro de la pantalla. Cada variación del objetivo es inicializada con la ubicación del cursor en el centro de la pantalla seguida de la asignación aleatoria de una de las metas. Las características de la prueba - p. ej., tamaño de las metas, distancia entre las metas, colores, número de intentos o cambio aleatorio de la meta para cada intento- fueron establecidas con la intención de brindar al usuario rangos de dificultad que fijen puntos de comparación para la evaluación del IMU-Mouse.

Se fija un número de dos sesiones de prueba compuestas cada una por 16 variaciones de la posición de la meta. La cantidad de sesiones es asignada teniendo en mente la reducción de los efectos de adaptación del usuario al procedimiento de la prueba. En cada variación se selecciona de manera aleatoria tres características de la meta: la posición en la pantalla, el ancho para todos los objetivos medido en píxeles el cual puede tomar un valor entre 75 o 100, y una distancia también medida en píxeles entre el centro de la pantalla y la nueva meta que puede cambiar entre 350 o 500. En la Fig. 5 se resume el proceso de selección de una meta. La meta se presenta al usuario en una de las excentricidades después de seleccionar con un clic el objetivo del centro de la pantalla. Cuando la meta es seleccionada satisfactoriamente, el objetivo en el centro aparecerá nuevamente siempre que no se hayan completado las 16 variaciones de la sesión de prueba actual.

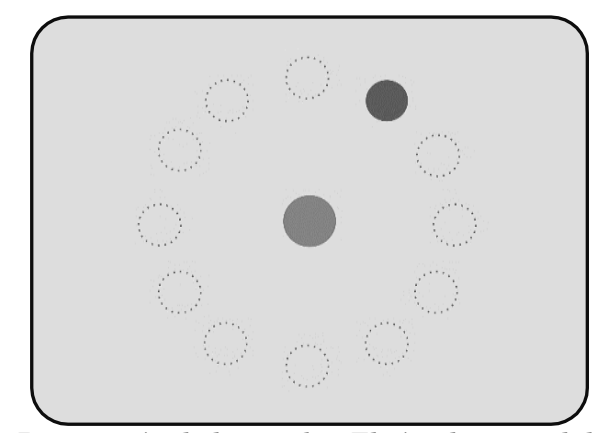

Fig. 5. Descripción de la prueba. El círculo central debe ser seleccionado mediante clic para que aparezca la meta. El círculo en la excentricidad representa la meta que debe ser seleccionada. Fuente: autor. 
IMU-Mouse: diseño e implementación de un dispositivo apuntador dirigido al desarrollo de interfaces adaptativas para personas con discapacidad física

Al finalizar las sesiones de prueba se solicita al usuario que responda una encuesta dirigida a la evaluación del IMUMouse. La construcción de la encuesta está basada en un cuestionario de evaluación de dispositivo sugerido en el anexo C del estándar ISO 9241-9. Las respuestas de cada participante fueron normalizadas mediante el uso de una escala de Likert de siete puntos, es decir, 7 es la calificación más favorable, 4 representa el punto medio, y 1 corresponde a la calificación menos favorable [31]. Al final del cuestionario son adicionadas dos preguntas con el fin de consultar el potencial que tiene el IMU-Mouse como alternativa para un usuario con discapacidad física, y para capturar las sugerencias que se podrían tener acerca del artefacto.

\section{RESULTADOS Y DISCUSIÓN}

En la primera parte de esta sección se valida el funcionamiento de las acciones de interacción del IMU-Mouse. La validación se hace con base en la captura de las señales del acelerómetro y del giroscopio mediante un programa desarrollado en Python que utiliza las librerías serial y pyqtgraph. La incorporación de la librería serial permite adquirir los datos enviados por la unidad de procesamiento. Estos son recibidos como una trama en la que se utiliza la coma como carácter de delimitación entre variables. El uso de la librería pyqtgraph permite la visualización en tiempo real de las señales de los sensores a través de una interfaz gráfica. El algoritmo identifica cada una de las variables y las presenta en un gráfico individual. En la segunda parte se analizan los resultados obtenidos a partir del experimento planteado en la sección anterior.

Además de la validación del funcionamiento, otra de las metas de esta sección es evidenciar la calidad de las señales utilizadas por el sistema. En cada una de las gráficas se observa que las señales son robustas para la discriminación de las diferentes acciones de interacción. Los valores del espacio de velocidad angular son relacionados de manera proporcional con un espacio que representa el desplazamiento en pantalla. La relación establece una nueva escala dirigida a excluir las variaciones en las medidas relacionadas con las componentes del ruido originadas por: sensibilidad cruzada entre los ejes, variaciones de posición inherentes al equilibrio y ruido propio de los dispositivos de medida. De esta manera, las señales utilizadas son robustas para el uso en este dispositivo. El cambio de espacio, realizado mediante la función map de Arduino, permite migrar del conjunto $\pm 2000 \%$ s con un tamaño de paso de $14.375 \mathrm{LSB} / \mathrm{s}$ al conjunto de valores enteros \pm 1360 con un incremento de 1. El tamaño del nuevo espacio de representación se fijó a partir la resolución del computador de la prueba en pro de la calidad de la interacción.

Las medidas $\left|a_{y z}\right|$ y $|\Delta \omega|$ son robustas para la discriminación de la acción de clic debido a su uso conjunto. Un $|\Delta \omega|$ cercano a $11 \%$ puede presentarse luego de un movimiento rápido de la cabeza. Sin embargo, la ocurrencia de dos picos de $\left|a_{y z}\right|$ y $|\Delta \omega|$ es característica de un evento de clic. Lo anterior implica que para esta aplicación no se hayan implementado métodos complementarios para el acondicionamiento de la señal -p. ej., algún tipo de filtro adicional-.

\subsection{Validación del diseño}

Las acciones de desplazamiento y clic ocurren cuando el dispositivo entra en estado de actividad. Este cambio de estado ocurre luego que se ha superado un valor de aceleración de $0.19 \mathrm{~g}$ en alguno de los ejes y o z. El mouse se desactiva cuando la aceleración cae por debajo de $0.13 \mathrm{~g}$ en los dos ejes mencionados. Cuando el sistema está en estado de inactividad, la información generada por el giroscopio no es tenida en cuenta. 
IMU-Mouse: diseño e implementación de un dispositivo apuntador dirigido al desarrollo de interfaces adaptativas para personas con discapacidad física

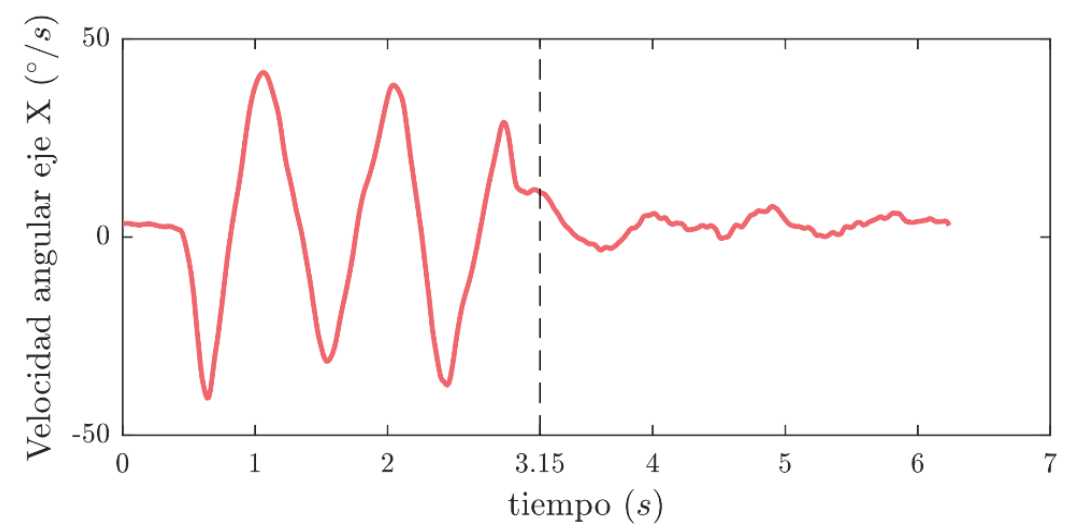

(a)

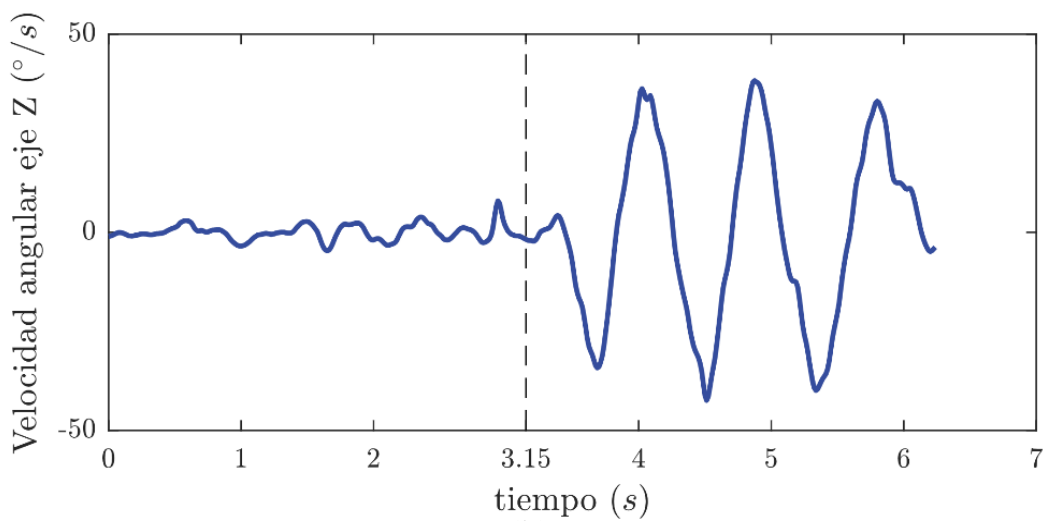

(b)

Fig. 6. Señales de velocidad angular en los ejes $\boldsymbol{x}$ y z. () En el lapso $\boldsymbol{t}=[\mathbf{0 , 3 . 1 5}]$ se ejecuta un movimiento vertical en pantalla.

(b) En el lapso $\boldsymbol{t}=[\mathbf{3 . 1 5}, \mathbf{6}]$ se ejecuta un desplazamiento horizontal en pantalla. Fuente: autor.

En Fig. 6 y 7 se presentan las señales tenidas en cuenta cuando son ejecutadas las acciones de desplazamiento y clic respectivamente.

En Fig. 6 se presentan las señales del giroscopio cuando el mouse está ejecutando la acción de desplazamiento. En Fig. 6.a se visualiza la onda generada por los datos obtenidos a partir del eje x cuando se mueve la cabeza sobre el eje longitudinal. Los datos de esta señal hacen que el cursor oscile repetidamente entre la parte superior e inferior de la pantalla. En Fig. 6.b se presenta la onda originada por los datos del eje $z$ cuando se mueve la cabeza sobre el eje transversal. Los datos hacen que el cursor oscile reiteradamente entre la izquierda y la derecha. Estas señales de velocidad angular son utilizadas para el movimiento del cursor en pantalla luego de ser de ser relacionadas proporcionalmente a un desplazamiento en pixeles. Esta relación permite disminuir la sensibilidad cruzada entre los ejes, es decir, un desplazamiento horizontal en pantalla cuando se mueve la cabeza sobre el eje longitudinal.

En Fig. 7 se muestran las señales construidas a partir de los datos del acelerómetro y el giroscopio cuando ocurre el clic. El proceso de detección inicia cuando la magnitud de la resultante de aceleración en los ejes $y$ e $z$ supera el valor de $1.09 \mathrm{~g}$ y el $|\Delta \omega|$ supera los $12 \%$ s. Luego de este evento se inicia un temporizador de $100 \mathrm{~ms}$ en donde se continúa calculando la resultante de la aceleración y el $|\Delta \omega|$. En caso de que en este lapso la magnitud resultante supere el valor de $1.08 \mathrm{~g}$ y el $|\Delta \omega|$ sea mayor o igual a $11 \%$, el dispositivo interrumpe el desplazamiento y efectúa la acción de clic. 
IMU-Mouse: diseño e implementación de un dispositivo apuntador dirigido al desarrollo de interfaces adaptativas para personas con discapacidad física

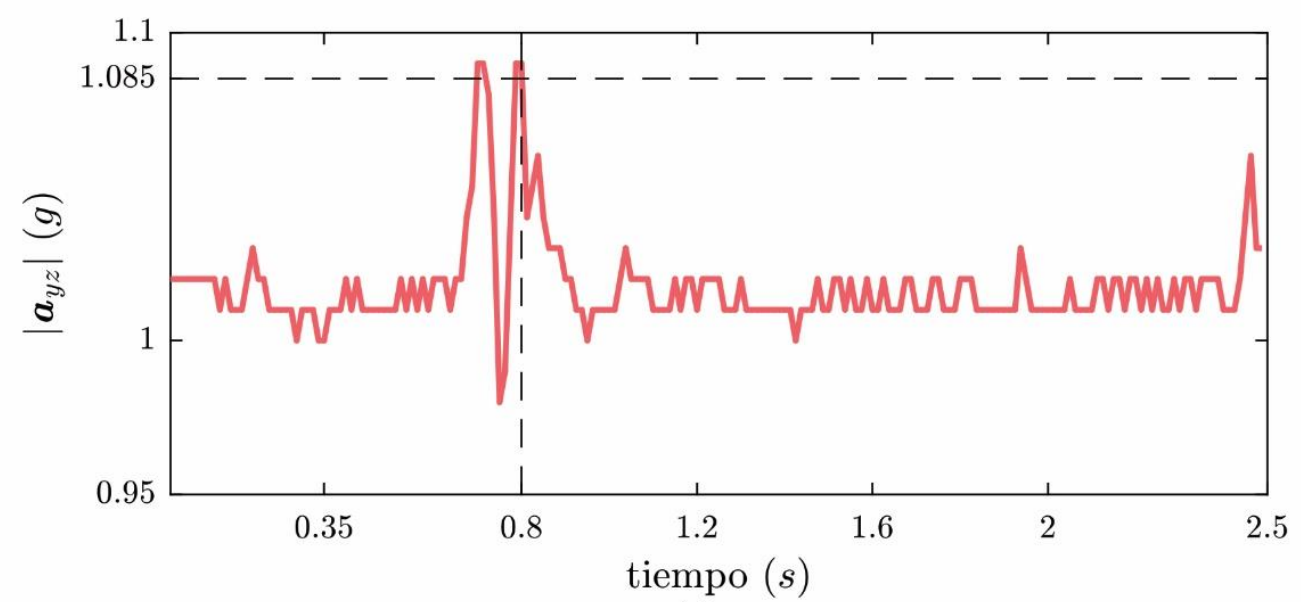

(a)

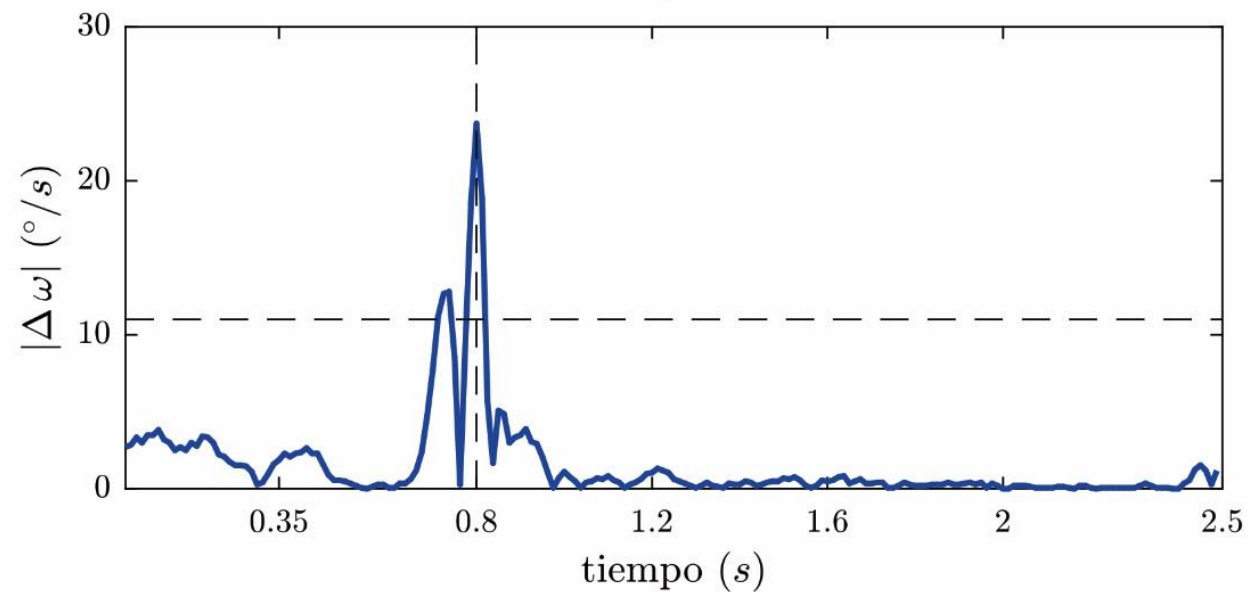

(b)

Fig. 7. Señales utilizadas para la determinación de la acción de clic, la acción ocurre en $t=0.8 \mathrm{~s}$. (a) Magnitud $\left|\boldsymbol{a}_{y z}\right|$. (b) Valor absoluto de la diferencia entre la muestra actual del giroscopio y la muestra anterior a esta $|\Delta \omega|$ en el eje x. Fuente: autor.

\subsection{Evaluación de la interacción}

En la Fig. 8 se presentan los resultados del cuestionario de evaluación del dispositivo IMU-Mouse mediante el uso de diagramas de caja y bigotes [31]. La percepción de los usuarios al comparar el IMUMouse frente al dispositivo apuntador usualmente utilizado, muestra que el primero habilita una interacción efectiva a través del cursor. El costo de la interacción para el cuerpo del individuo es observado en el valor de las medias por debajo de la mitad de la escala en lo que respecta a la fatiga en cuello y cabeza. Lo anterior se explica porque las acciones de desplazamiento y clic dependen de los cambios de velocidad angular y aceleración generados por el cuello y la cabeza. Sin embargo, el confort general durante la interacción -en donde son incluidos los hombros- se percibe como favorable por parte de los participantes. En la figura también se indica que no es requerido un nivel elevado de esfuerzo físico y mental para operar el IMU-Mouse.

En lo que respecta a las acciones de interacción, se observa que los participantes manifiestan una percepción favorable frente a algunas de sus características. 
IMU-Mouse: diseño e implementación de un dispositivo apuntador dirigido al desarrollo de interfaces adaptativas para personas con discapacidad física

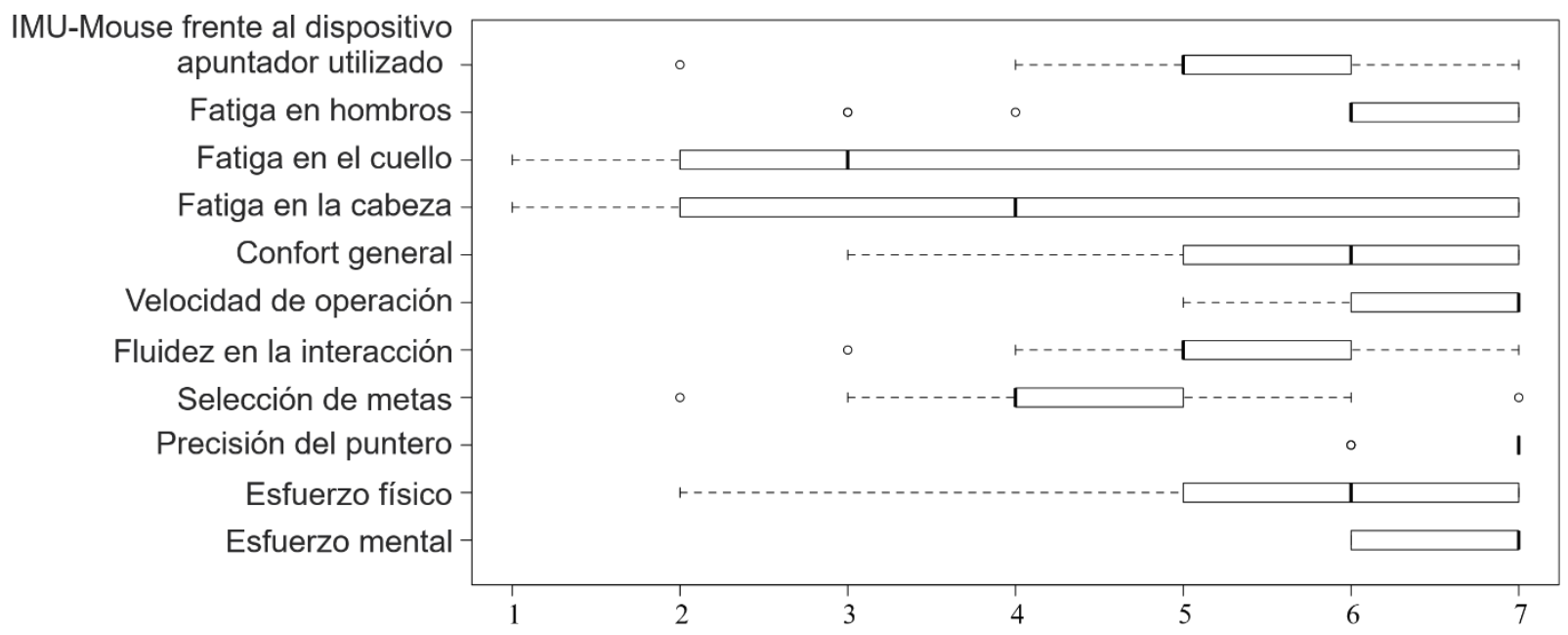

Fig. 8. Cuestionario de evaluación del dispositivo IMU-Mouse. El valor de 7 indica la respuesta más favorable y 1 indica la respuesta menos favorable. Los círculos fuera de los diagramas de caja representan puntos atípicos. Fuente: autor.

La media de siete obtenida en la velocidad de operación se debe a que los retardos necesarios para la ejecución de las acciones no causaron incomodidad. Por el contrario, la percepción acerca de la calidad de la fluidez en la interacción y la selección de las metas decrece en comparación con la característica anterior. La disminución se justifica porque estas dos características dependen de la precisión de una acción de clic que no es independiente a la acción de desplazamiento. La precisión del puntero fue la característica mejor ponderada y se explica porque el desplazamiento del cursor hasta cualquier lugar de la pantalla no presentó dificultades.

En las preguntas abiertas acerca del potencial del IMU-Mouse ante la discapacidad, los participantes señalaron que el dispositivo constituye una herramienta adecuada para asistir la interacción. La razón de este señalamiento tiene como base el desempeño del aparato durante la prueba y su capacidad de ser operado sin los brazos. Al ser indagados por la calidad de la interacción, los usuarios se mostraron conformes debido a que fueron capaces de realizar las tareas propuestas. Sin embargo, las opiniones recibidas respecto al procedimiento implementado para la ejecución del clic invitan a su mejoramiento. Entre las observaciones recibidas se destacan: el cansancio inducido por el cambio de aceleración que requieren el clic y la transición al estado de actividad, las molestias en el cuello ante la dificultad de seleccionar las metas ubicadas en las esquinas, y el desajuste de la diadema.

\section{CONCLUSIONES}

Uno de los objetivos que buscaba la ejecución de la encuesta es la consulta acerca de la capacidad del IMU-Mouse para desempeñarse como un dispositivo apuntador. La conclusión obtenida permite afirmar que el diseño e implementación del dispositivo es efectivo para interactuar mediante las acciones de desplazamiento del cursor y clic. Asimismo, la efectividad en la interacción está sustentada en las respuestas obtenidas respecto a la calidad y en las observaciones de los investigadores durante la prueba. A pesar de que el IMU-Mouse permitió la interacción, los usuarios hicieron las siguientes sugerencias: afinar la transición del reposo al estado de actividad, hacer que el dispositivo sea más liviano, dotar al dispositivo de una 
IMU-Mouse: diseño e implementación de un dispositivo apuntador dirigido al desarrollo de interfaces adaptativas para personas con discapacidad física

acción de reinicio que ubique el cursor en la mitad de la pantalla, construir un mecanismo para ajustar la diadema, y dotar al dispositivo de comunicación inalámbrica.

Después de la prueba, también se concluye que existen espacios para la implementación de complementos de hardware y software en el IMU-Mouse. Los complementos tienen como objetivo obtener variaciones para la adaptación de interfaces desde la capa de interacción. A diferencia de las sugerencias reportadas en el párrafo anterior, los complementos buscan enriquecer la interacción mediante la intervención directa sobre la ejecución de las acciones tal como el clic. Los espacios para su aplicación serán expresados en función de las siguientes limitaciones: dificultad para seleccionar los objetivos de las esquinas, cansancio inducido por el golpeteo del clic, y falta de precisión del clic debido a su dependencia con la acción de desplazamiento. Las limitaciones permiten concluir que los complementos deberán representar alternativas para la ejecución del clic.

Como trabajo futuro se propone la implementación de los complementos para la construcción de variantes del IMU-Mouse, la comparación del dispositivo y sus variantes con otras propuestas, el desarrollo de mejoras encaminadas a atender las sugerencias realizadas por los participantes de la prueba entre la que se encuentra el diseño de una nueva diadema, y el mejoramiento de la calidad de las señales de los sensores mediante el uso de filtros.

\section{AGRADECIMIENTOS}

Este trabajo fue realizado bajo las subvenciones de las siguientes fuentes: el proyecto financiado por la Universidad Nacional de Colombia con código Hermes: 23169, y el proyecto COLCIENCIAS RAIM con código 1119-569-34172.

\section{REFERENCIAS}

[1] M. Peissner, D. Häbe, and A. Schuller, "MyUI: Mainstreaming Accessibility through Synergistic User Modelling and Adaptability," The University of Lowa, 2011.

[2] K. Hinckley, "Input Technologies and Techniques," in The Human-Computer Interaction Handbook: Fundamentals, Evolving Technologies and Emerging Applications, 2nd ed., A. Sears and J. A. Jacko, Eds. New York: Human Factors and Ergonomics, 2009, pp. 161-172.

[3] C. Fichten, M. Barile, J. V Asuncion, and M. Fossey, "What government, agencies, and organizations can do to improve access to computers for postsecondary students with disabilities: Recommendations based on Canadian empirical data," Int. J. Rehabil. Res., vol. 23, no. 3, pp. 191-199, 2000.

[4] K. Z. Gajos, D. S. Weld, and J. O. Wobbrock, "Automatically generating personalized user interfaces with Supple," Artif. Intell., vol. 174, no. 12-13, pp. 910-950, Aug. 2010.

[5] B. Phillips and H. Zhao, "Predictors of Assistive Technology Abandonment," Assist. Technol., vol. 5, no. 1, pp. 36-45, Jun. 1993.

[6] K. Giotopoulos, C. Alexakos, G. Beligiannis, and A. Stefani, "Bringing AI to E-learning," Int. J. Inf. Commun. Technol. Educ., vol. 6, no. 2, pp. 24-35, 2010.

[7] P. A. Rodríguez, N. D. Duque, and D. A. Ovalle, "Método Híbrido de Recomendación Adaptativa de Objetos de Aprendizaje basado en Perfiles de Usuario," Form. Univ., vol. 9, no. 4, pp. 83-94, 2016.

[8] P. Brusilovsky and E. Millán, "User Models for Adaptive Hypermedia and Adaptive Educational Systems," in The Adaptive Web, P. Brusilovsky, A. Kobsa, and W. Nejdl, Eds. Berlin, Heidelberg: Springer Berlin Heidelberg, 2007, pp. 3-53.

[9] N. D. Duque Méndez, "Modelo Adaptativo Multi-Agente para la Planificación y Ejecución de Cursos Virtuales Personalizados," Universidad Nacional de Colombia, 2009.

[10] T. C. Davies, S. Mudge, S. Ameratunga, and N. S. Stott, "Enabling self-directed computer use for individuals with cerebral palsy: a systematic review of assistive devices and technologies," Dev. Med. Child Neurol., vol. 52, no. 6, pp. 510-516, Jun. 2010.

[11] D. W. K. Man and M. S. L. Wong, "Evaluation of Computer-Access Solutions for Students With Quadriplegic Athetoid Cerebral Palsy," Am. J. Occup. Ther., vol. 61, no. 3, pp. 355-364, May 2007.

[12] C. Kouroupetroglou, Enhancing the Human 
IMU-Mouse: diseño e implementación de un dispositivo apuntador dirigido al desarrollo de interfaces adaptativas para personas con discapacidad física

Experience through Assistive Technologies and E-Accessibility, Hershey, PA: IGI Global, 2014.

[13] L. C. Barrera, H. Loaiza, and S. E. Nope, "Comando de un cursor en 2-D mediante señales EEG," Entre Cienc. e Ing., no. 15, pp. 24-31, 2014.

[14] X. Huo, H. Park, J. Kim, and M. Ghovanloo, "A Dual-Mode Human Computer Interface Combining Speech and Tongue Motion for People with Severe Disabilities," IEEE Trans. Neural Syst. Rehabil. Eng., vol. 21, no. 6, pp. 979-991, 2013.

[15] C. A. Castillo Benavides, L. F. García, N. D. Duque Méndez, and J. H. Estrada Estrada, "Mouse para personas con discapacidad física en contextos educativos," Rev. Ing. $e$ Innovación, vol. 3, no. 1, pp. 82-89, 2015.

[16] Y. wook Kim and J. hyun Cho, "A novel development of head-set type computer mouse using gyro sensors for the handicapped," in 2nd Annual International IEEE-EMBS Special Topic Conference on Microtechnologies in Medicine and Biology., 2002, pp. 356-359.

[17] N. Nakazawa, K. Yamada, T. Matsui, and I. Itoh, "Development of welfare supportequipment for personal computer operation with head tilting and breathing," in 31st Annual Conference of IEEE Industrial Electronics Society, 2005. IECON 2005., 2005, pp. 201-206.

[18] C. Gerdtman and M. Lindén, "Development of a gyro sensor based computer mouse with usb interface as technical aid for disabled persons," in 3rd European Medical \& Biological Engineering Conference, 2005, pp. $1-5$.

[19] M. Bureau, J. M. Azkoitia, G. Ezmendi, I. Manterola, H. Zabaleta, M. Perez, and J. Medina, "Non-Invasive, Wireless and Universal Interface for the Control of Peripheral Devices by Means of Head Movements," in 10th International Conference on Rehabilitation Robotics, 2007, pp. 124-131.

[20] T. Simpson, C. Broughton, M. J. A. Gauthier, and A. Prochazka, "Tooth-click control of a hands-free computer interface.," IEEE Trans. Biomed. Eng., vol. 55, no. 8, pp. 20506, Aug. 2008.
[21] R. Raya, J. O. Roa, E. Rocon, R. Ceres, and J. L. Pons, "Wearable inertial mouse for children with physical and cognitive impairments," Sensors Actuators A Phys., vol. 162 , no. 2 , pp. 248-259, Aug. 2010.

[22] S. Kim, M. Park, S. Anumas, and J. Yoo, "Head mouse system based on gyro and opto sensors," in 3rd International Conference on Biomedical Engineering and Informatics, 2010, pp. 1503-1506.

[23] N. Sim, C. Gavriel, W. W. Abbott, and A. A. Faisal, "The Head Mouse - Head Gaze Estimation 'In-the-Wild' with Low-Cost Inertial Sensors for BMI Use," in 6th International IEEE/EMBS Conference on Neural Engineering (NER), 2013, pp. 735738.

[24] W. H. Baird, "An introduction to inertial navigation," Am. J. Phys., vol. 77, no. 9, pp. 844-847, Sep. 2009.

[25] N. Yazdi, F. Ayazi, and K. Najafi, "Micromachined inertial sensors," Proc. IEEE, vol. 86, no. 8, pp. 1640-1659, 1998.

[26] S. Reif Acherman, "Juguetes como Instrumentos de Enseñanza en Ingeniería: Los casos del Péndulo de Newton y el Giroscopio," Ing. y Compet., vol. 16, no. 2, pp. 189-198, 2014.

[27] Y. S. Ryu, D. H. Koh, D. Ryu, and D. Um, "Usability Evaluation of Touchless Mouse Based on Infrared Proximity Sensing," J. Usability Stud., vol. 7, no. 1, pp. 31-39, 2011.

[28] X. Zhang and I. S. MacKenzie, "Evaluating Eye Tracking with ISO 9241 - Part 9," in Human-Computer Interaction. HCI Intelligent Multimodal Interaction Environments, J. A. Jacko, Ed. Berlin, Heidelberg: Springer Berlin Heidelberg, 2007, pp. 779-788.

[29] International Organization for Standardization, "Ergonomic requirements for office work with visual display terminals (vdts) - part 9: requirements for nonkeyboard input devices," ISO 92419:2000(en), 2012.

[30] R. Likert, "A technique for the measurement of attitudes," Arch. Psichol., vol. 22, no. 140, pp. 5-55, 1932.

[31] J. W. Tukey, Exploratory data analysis. Pearson, 1977. 